\title{
Thermal Radiative Simulations and Measurements of a Scramjet Test Rig
}

\author{
Andrew J. Crow* and Iain D. Boyd \\ University of Michigan, Ann Arbor, Michigan 48109 \\ Michael S. Brown" \\ U.S. Air Force Research Laboratory, Wright-Patterson Air Force Base, Ohio 45433 \\ and \\ Jiwen Liů̊
}

Taitech, Inc., Wright-Patterson Air Force Base, Ohio 45433

DOI: $10.2514 / 1 . B 35207$

\begin{abstract}
Thermal radiation is a poorly understood process in scramjet engines, but it may play a significant role in the flow and wall heating of the combustion chamber. However, current simulation methods for predicting the thermal radiation in a flight scramjet combustion chamber have yet to be validated with experiments. An experimental measurement apparatus is placed at the rear exit of the HIFiRE 2 direct-connect rig at NASA's Langley Research Center. An array of photodetectors gather emission in the infrared along several lines of sight across the combustor exit. The fields of view are simulated using a ray-tracing-method program that postprocesses a computational fluid dynamics flowfield simulation of the test rig. The ray-tracing program employs a simplified two-point correlated- $k$ spectral model with spectral model error bars. The predictions show an overlap in sensor and experimental predictions for 13 of 16 photodetectors.
\end{abstract}

\begin{tabular}{|c|c|c|}
\hline & & Nomenclature \\
\hline$A$ & $=$ & area, $\mathrm{m}^{2}$ \\
\hline$a$ & $=$ & spectral quadrature weight \\
\hline$B_{\nu}$ & $=$ & blackbody intensity, $\mathrm{W} /\left(\mathrm{m}^{2} \cdot \mathrm{Hz} \cdot \mathrm{sr}\right)$ \\
\hline$c_{1}$ & $=$ & tuning parameter \\
\hline$E$ & $=$ & estimated error \\
\hline$F$ & $=$ & spectrally integrated heat flux, $\mathrm{W} / \mathrm{m}^{2}$ \\
\hline$F_{\nu}$ & $=$ & spectrally specific heat flux, $\mathrm{W} /\left(\mathrm{m}^{2} \cdot \mathrm{Hz}\right.$ \\
\hline$f_{\nu}$ & $=$ & scattering redistribution function \\
\hline$I_{\nu}$ & $=$ & radiative intensity, $\mathrm{W} /\left(\mathrm{m}^{2} \cdot \mathrm{Hz} \cdot \mathrm{sr}\right)$ \\
\hline$i$ & $=$ & location index \\
\hline$j$ & $=$ & ordinate index \\
\hline$k$ & $=$ & frequency index \\
\hline$l$ & $=$ & spectral quadrature index \\
\hline$N$ & $=$ & maximum index number \\
\hline$n$ & $=$ & species index \\
\hline$S_{\nu}$ & $=$ & spectral line strength, $1 / \mathrm{m}$ \\
\hline$s$ & $=$ & trace location, m \\
\hline$T$ & $=$ & temperature, $\mathrm{K}$ \\
\hline$U$ & $=$ & spectral uncertainty \\
\hline$w$ & $=$ & standoff position, m \\
\hline$X$ & $=$ & mole fraction \\
\hline$x$ & $=$ & streamwise position, $\mathrm{m}$ \\
\hline$y$ & $=$ & vertical position, $\mathrm{m}$ \\
\hline$z$ & $=$ & spanwise position, $\mathrm{m}$ \\
\hline$\theta$ & $=$ & azimuthal angle, rad \\
\hline
\end{tabular}

Received 10 October 2013; revision received 24 March 2014; accepted for publication 24 March 2014; published online 27 June 2014. This material is declared a work of the U.S. Government and is not subject to copyright protection in the United States. Copies of this paper may be made for personal or internal use, on condition that the copier pay the $\$ 10.00$ per-copy fee to the Copyright Clearance Center, Inc., 222 Rosewood Drive, Danvers, MA 01923; include the code 1533-3876/14 and \$10.00 in correspondence with the CCC.

*Currently Research Scientist, CRAFT-Tech, Inc.; acrow@ craft-tech.com. Member AIAA.

†Professor; iainboyd@umich.edu. Fellow AIAA.

Physicist, Aerospace Propulsion Division; michael.brown@ wpafb.af.mil. Associate Fellow AIAA.

${ }^{\S}$ Senior Research Scientist, Aerospace Propulsion Division; jiwen.liu.ctr@ wpafb.af.mil. Associate Fellow AIAA.

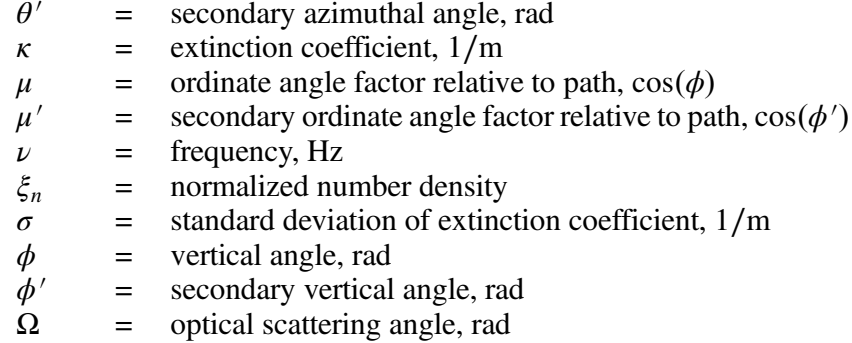

Subscripts

$\begin{array}{ll}\text { band } & =\text { number of frequency bands } \\ \text { filt } & =\text { quantity at filter } \\ \text { LBL } & =\text { line-by-line variable } \\ \max & =\text { domain upper limit } \\ \min & =\text { domain lower limit } \\ \text { quad } & =\text { correlated- } k \text { quadrature scheme } \\ \text { sens } & =\text { quantity at sensor } \\ \text { spec } & =\text { number of species } \\ \nu & =\text { frequency-specific value } \\ 0 & =\text { nominal state }\end{array}$

\section{Introduction}

$\mathbf{T}$ HERE is a desire to operate scramjet engines at the highest possible temperature in order to maximize the thrust that can be generated. The role of thermal radiation in the energy balance inside a combustor has been little studied and is poorly understood. Previous work studied the hydrogen-fueled HyShot II combustion chamber and found the thermal radiation to be insignificant [1]. The current work considers combustion chambers similar to the Hypersonic International Flight Research Experiment 2 (HIFiRE 2) supersonic combustor [2-4]. It has been demonstrated that the radiative component of wall heat flux may be higher in the HIFiRE 2 combustor due to two effects: 1) its use of hydrocarbon fuel that generates more significant quantities of radiating species such as carbon dioxide that are absent from HyShot II, and 2) the HIFiRE 2 combustor is significantly larger in volume than that for HyShot II $[\underline{5}, 6]$. 
Radiation is evaluated using a two-step approach. First, the combustion flow is computed using a turbulent Reynolds-average Navier-Stokes (RANS) computational fluid dynamics (CFD) code. These computations were already presented in previous work by the U.S. Air Force Research Laboratory (AFRL) [4,5]. Second, the flowfield results are used as input into radiative heat transfer simulations that simulate the radiative heat transfer intercepted by the photodetectors used on the experimental test rig. The current work uses a ray-tracing technique with a spectrally resolved narrow-band approximation model. The narrow-band spectral approximation introduces inherent epistemic error from both the source databases used as well as from the approximations made in creating banded models $[7,8]$. The propagation of these errors through the radiation predictions are analyzed to place uncertainty bounds on the computed results.

The spectrally resolved thermal radiation results with their associated errors are compared to a series of experimental measurements taken at NASA's Langley Research Center on the HIFiRE direct-connect rig (HDCR) [9]. The experimental measurements are made with 16 photodetectors placed at the outflow of the HDCR viewing the flow through a protective aperture. The radiative simulations attempt to duplicate this field of view, and the results can be directly compared. Direct thermal radiative measurements have been performed in the past for scramjet thermal radiation, but these measurements have never been performed on a test rig directly duplicating a flight experiment $[10,11]$.

In the following sections, the CFD and radiation methodologies are described. The scramjet combustor rig and the radiation measurements are discussed. Results for the comparisons between the predicted and measured radiation emissions are presented. The paper ends with a summary and conclusions.

\section{Methodology}

The three-dimensional flowfield simulations are computed using a 22-species chemically reacting $k-\epsilon$ RANS CFD computation $[\underline{12}, 13]$. Previous work has presented these simulations comparing net thermal radiative heating and convective radiative heating $[\underline{5}, 6]$.

The resulting flowfield calculations are postprocessed to evaluate radiation effects using a three-dimensional ray-tracing (RT) method $[14,15]$. The three-dimensional discretized ray-tracing method provides a second-order spatial solution with a simplified correlated$k(\mathrm{C}-k)$ narrow-band spectral model. This method allows for both spatially and spectrally resolved solutions for radiation intensity, heat flux, absorptivity, and optical depth. However, it only solves for one location at a time.

The singular locations of the radiative heating measurements are employed to compare simulated thermal radiative heating predictions with experimental measurements taken from photodetectors on the HDCR. The photodetectors measure the direct infrared emission from the flowfield at the exit plane of the HDCR as viewed through a protective aperture. Previous works have partially addressed the infrared emissions of the HDCR through uncalibrated relative measurements and partially calibrated measurements [므, $\underline{6}]$.

\section{A. General Radiative Analysis}

For the three-dimensional thermal radiative analysis, the final CFD solution is projected onto a series of ray paths for converging at each location of interest. The total spectrally resolved radiative energy flux $F_{\nu}$ to a cell face is found by integrating the total angular intensity $I_{\nu}$ contributions that are normal to the plane of the cell face. This expression is given in Eq. (1), where $\mu$ is the cosine of the angle $\phi$ between the ordinate and the vector normal to the plane. The coordinate system is illustrated in Fig. 1:

$$
F_{\nu}(s)=\int_{0}^{2 \pi} \int_{0}^{1} I_{\nu}(s, \mu, \theta) \mu \mathrm{d} \mu \mathrm{d} \theta
$$

The main equation for radiative intensity is the radiative transfer equation, which is given by Eq. (2), in which the left-hand side of the equation represents the change in intensity over distance and the

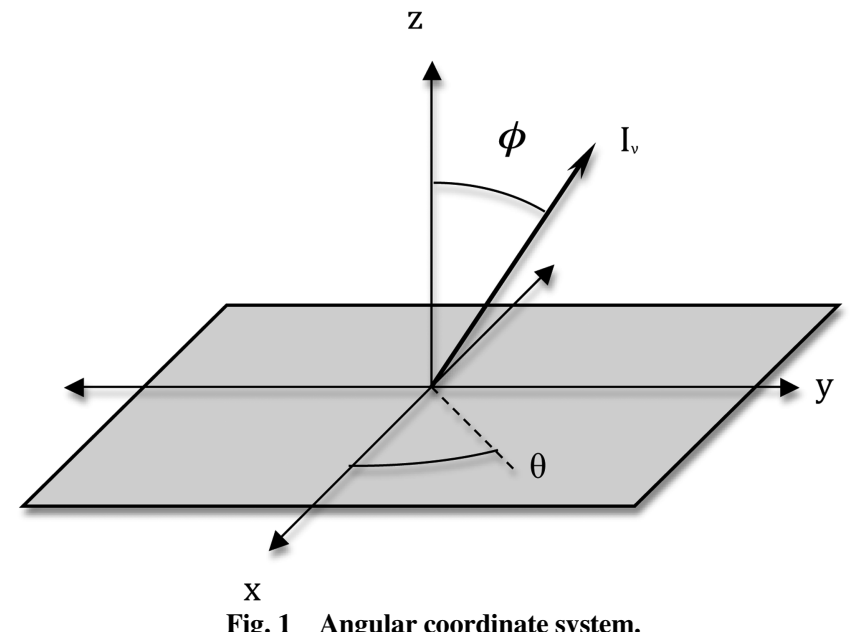

Fig. 1 Angular coordinate system.

terms on the right-hand side represent, from left to right, extinction due to absorption, extinction due to scattering, contribution due to scattering, and contribution due to emission. The term $f_{\nu}$ refers to the scattering redistribution function in which the source angles (the secondary angles) are denoted by primes. The term $B_{\nu}$ denotes the frequency-specific blackbody intensity as determined by the Planck distribution function [16]:

$$
\begin{aligned}
& \frac{\mathrm{d} I_{\nu}(s, \mu, \theta)}{\mathrm{d} s}=-\kappa_{\mathrm{abs}}(s) I_{\nu}(s, \mu, \theta)-\kappa_{\mathrm{sca}}(s) I_{\nu}(s, \mu, \theta) \\
& \quad+\frac{\kappa_{\mathrm{sca}}(s)}{4 \pi} \int_{0}^{2 \pi} \mathrm{d} \theta^{\prime} \int_{-1}^{1} \mathrm{~d} \mu f_{\nu}\left(s, \mu, \theta, \mu^{\prime}, \theta^{\prime}\right) I_{\nu}\left(s, \mu^{\prime}, \theta^{\prime}\right) \\
& +\kappa_{\mathrm{abs}}(s) B_{\nu}(T(s))
\end{aligned}
$$

The boundary conditions at the walls are modeled as completely absorbing nonemissive walls.

The high temperatures in scramjet engines prevent fuel or water droplet formation, meaning that Mie scattering is not possible. Additionally, the wavelengths considered are in the infrared regime, which is not significantly affected by Rayleigh scattering [17]. Soot formation has not been observed in any optical measurement, of the HDCR, and thus its scattering effects can be ignored. Since all forms of particle scattering are not significant in this case, it will be ignored in all analysis $\left(\kappa_{\mathrm{sca}}=0\right)$.

\section{B. Ray-Tracing Method for Radiative Analysis}

A second-order spatially discretized three-dimensional ray-tracing method with a two-point narrow-band spectral method is employed to simulate the thermal radiation within the HDCR [15]. Whereas the selection of paths for the ray-tracing method is often chosen with a Monte Carlo scheme, a predetermined ray spacing is opted for in this application [14]. The ray-tracing method divides a solid-angle hemisphere into a discrete series of ordinates, each with an assigned three-dimensional direction. The radiative heat transfer is integrated along a single spatial direction for each ordinate until it reaches a simulation boundary. The one-dimensional path integration is calculated with an implicit second-order backward-differencing scheme as given by Eq. (3). The individual intensities are then integrated angularly as in Eq. (1), which can be performed numerically with Eq. (ㅁ) [모]:

$$
\frac{3 I_{\nu}(i, j)-4 I_{\nu} 0(i-1, j)+I_{\nu} 0(i-2, j)}{2 \Delta s(i)}=\kappa_{a b s}(i)\left(B_{\nu}(i)-I_{\nu}(i, j)\right)
$$

$$
F_{\nu}=\sum_{j}^{N_{\text {ord }}} I_{\nu}(\theta(j), \phi(j)) \sin (\phi(j)) \cos (\phi(j)) \sin (\Delta \phi(j)) \Delta \theta(j)
$$




\section{Spectral Model}

The absorption coefficients for the ray-tracing method are determined by a simplified two-point narrow-band C- $k$ spectral model. Whereas detailed multispecies $\mathrm{C}-k$ methods are very accurate, they can require large amounts of computational time to generate all of the cell-specific quantities. This time can be reduced by using precomputed spectral tables, but these tables can be large to the point of being unusable in a realistic computational system [18]. Many simplifications to the $\mathrm{C}-k$ method have been developed to compromise between accuracy, time, and memory requirements $[19,20]$. The method chosen for this study assumes a narrow-band two-point Gaussian C- $k$ distribution.

All frequency-based models assume that the total flux is calculated by a frequency integration of the spectrally resolved flux as given in Eq. (5):

$$
F(s)=\int_{\nu_{\min }}^{\nu_{\max }} F_{\nu}(s) \mathrm{d} \nu
$$

Realistic mixed-gas spectra are very complicated, with hundreds of millions of spectral lines that depend on temperature, pressure, and species concentration. The line-by-line method allows for the arbitrarily fine computation of the spectral absorptivity at any frequency $[14,20]$. Narrow-band methods divide the absorption spectrum into bands of given width. In this case, $25-\mathrm{cm}^{-1}$-wide bands are employed. Prior works have used narrow-band selections ranging from 5- to $250-\mathrm{cm}^{-1}$-wide spectral bands $[18,21]$. Generalized absorption characteristics are then calculated for each band using the arbitrarily fine absorption spectrum as calculated by the line-by-line method. The C- $k$ method transforms the very fine resolution absorption spectrum from the line-by-line method into an ordered monotonic absorption spectrum, which can be approximated with a handful of quadrature points as in Eq. (6), where $a$ is the quadrature weight, $l$ is the quadrature index, and $k$ is the spectral band index. The extreme simplification of this approach is to take a quadrature point at the statistical average of the absorption band and set the quadrature weight equal to 1 for the single point in the band [1]:

$$
F(s)=\sum_{k}^{N_{\text {band }}} \sum_{l}^{N_{\text {quad }}} a(l) F_{\nu}(s, k, l) \Delta \nu(k)
$$

A step up from a band-averaged approach is to choose two equally weighted quadrature points $(a=0.5)$ at 1 standard deviation above and below the mean band absorptivity. A true C- $k$ method would require the reordering of the absorption spectrum of each band and numerically calculating the weighted value for both standard deviation locations. A simplified method is chosen for the current work, which will maintain the first moments of the absorption distribution within a given spectral band. The reasoning behind the simplified method is that it will approach the accuracy of a line-byline calculation in the optically thin limit. The way to guarantee this is to always maintain the weighted average of all absorption coefficients as equal to the average absorption (first moment) as calculated by a line-by line extraction as given in Eq. (7):

$$
\sum_{l}^{N_{\text {quad }}} a(l) \kappa_{\nu}(l)=\int_{\nu_{\min }}^{\nu_{\text {man }}} \kappa_{\mathrm{LBL}}(\nu) \mathrm{d} \nu / \int_{\nu_{\min }}^{\nu_{\text {man }}} \nu \mathrm{d} \nu
$$

A simplified method to maintain the second moment involves setting the quadrature points to values of the standard deviations of the distribution. These can be maintained if an error function is employed, as in Eq. (8) where $\bar{\kappa}$ is the band-averaged absorption as calculated from Eq. (7) when the number of quadrature points $N_{\text {quad }}$ is equal to 1 . The standard deviation of the line-by-line extraction of for each band is given by $\sigma$. An accuracy tuning parameter also exists in Eq. ( ) and is given by $c_{1}$, which is chosen to improve the accuracy of the model over test unit problems without affecting the value of $\bar{\kappa}$ :

$$
\kappa_{\nu}(s, l)=\bar{\kappa}_{\nu}(s)\left(1+\operatorname{ERF}\left(\frac{ \pm \sigma_{\nu}(s)}{\bar{\kappa}_{\nu}(s)}\right) c_{1}\right)
$$

The choice of using an error function distribution instead of a true inversion is because the error function is symmetric about the mean value. Thus, as long as the quadrature points are symmetrically weighted and spaced about the mean, then the value of $\bar{\kappa}$ will be maintained for any distribution. The choice of $25 \mathrm{~cm}^{-1}$ over the spectral range of interest remains to be optimized for the bandwidth and number of quadrature points, and quadrature weighting. Future modeling will attempt to find the band parameters balancing both accuracy and computational expense.

All spectral bands are assumed to be independent with no frequency interacting with another. Thus, the total integration can be computed as the sum of the spectrally resolved flux multiplied by the bandwidth for each frequency as in Eq. (6).

However, there is a question as to whether or not within a band the individual quadrature points have any cross-correlation. A simplified approach is taken in the current model. The quadrature absorption values are assumed to have no cross-correlation with the other quadrature points. Thus, each quadrature point of each band can be solved for independently.

The calculation of absorption coefficients is performed with a lineby-line calculation for all species of interest at representative pressures and temperatures pertinent to the conditions inside a scramjet. The method of calculating the absorption coefficients is presented in previous works, and the spectral methods and accuracy are examined in greater detail in the thesis by Crow et al. $[5,6]$. The individual lines are extracted from the HiTemp database using JAVAHAWKS at a pressure of $2 \mathrm{~atm}$ and temperatures of 500, 1000, $1500,2000,2500$, and $3000 \mathrm{~K}$, respectively [7]. The data are extracted for water vapor, carbon dioxide, carbon monoxide, and the hydroxyl radical. The individual lines are processed at regular intervals for the summation of Lorentz line profiles using the procedure laid out in Sec. II.3 of the thesis by Irvine [22]. In the procedure, the line strength and other pertinent data are extracted from the HiTemp database using a given partition function. For the case of this work, the partition functions are those inherent to the JAVAHAWKS software. The extracted line information is then given a Lorentz line profile, and the sum effect of these profiles is calculated for all of the lines in a species for each frequency of interest. The frequencies of interest range from 25 to $10,000 \mathrm{~cm}^{-1}$ at even intervals. These calculations are repeated for each species of interest. The extracted data are used to calculate a series of optical depths for each species at each temperature with a spectral resolution of $0.01 \mathrm{~cm}^{-1}$, which, in turn, is used to create the band statistic needed for the spectral model, resulting in a series of fast spectral lookup tables for each species. The lookup tables can be used to directly evaluate the species absorption as in Eq. (9), where $n$ denotes the species index, $S_{\nu}$ denotes the species absorptivity extracted from the lookup table, $X(i, n)$ represents the mole fraction, and $\xi_{n}(i)$ represents the relative number density normalized to the number densities used to calculate the lookup tables. With this process in place, the absorption coefficient can quickly be calculated for any combination of species concentration mixtures at a wide range of temperatures. If the raw extracted data are used directly, assuming each point is a band with one quadrature point, then they can be treated as a very high spectral fidelity lookup table:

$$
\kappa_{\nu}(i, l)=\sum_{n}^{N_{\mathrm{spec}}} S_{\nu}(T(i), n, l) X(i, n) \xi_{n}(i)
$$

\section{Error Propagation}

With the use of a spectral model, a means of estimating the uncertainty from the absorption coefficients stemming from the spectral tables becomes necessary, as does predicting the error from the modeling approximation. Comparisons between high- and lowfidelity spectral models were additionally performed in previous works $[\underline{6}, \underline{22}]$. The simplest means of determining the possible error 
contributions is a differencing scheme. To estimate the modeling error, a one-dimensional unit problem is employed. The problem compares a high spectral resolution and a low spectral resolution calculation of the total heat flux within each band as given by Eq. (6). The high-resolution band model has 2500 equally spaced bands for every $25 \mathrm{~cm}^{-1}$ in the spectrum. The model has identical resolution to the extracted line-by-line data described in the previous section. The reason these data are not refereed to as a line-by-line method is because they are not reextracted for every temperature or species partial pressure. Instead, the values are interpolated between the temperatures used for the initial data extraction, and the species contributions are accounted for as described in the previous section. These bands have a single-quadrature point band taken directly from a line-by-line sampling. The low-resolution band employs two quadrature points per band as calculated by Eq. (8). The results of these two methods are compared in order to calculate the error as given in Eq. (10), where $E_{\nu}$ represents the band-specific dimensionless error. Additionally, a factor of experimental uncertainty from the HiTemp databases is added in Eq. (10) as E. Since each individual line of the HiTemp database has its own associated experimental uncertainty, a general assumption of $E=20 \%$ is employed for all bands for which previous studies have estimated the database and Lorentz line error for water from the HiTemp database as given by Irvine [그,22]:

$$
E_{\nu}(k)=\operatorname{abs}\left(\frac{F_{\nu, \text { low }}(k)}{F_{\nu, \text { high }}(k)}\right)+E
$$

The unit problem is homogeneous, which means that fluxes for each quadrature point can be calculated using Beer's law as shown in Eq. (11), as opposed to a more complicated discrete-ordinates or ray-tracing method [17]:

$$
F_{\nu}(k, l)=B_{\nu}(T, k, l)\left(1-e^{-\kappa_{\nu}(k, l) \Delta s}\right)
$$

The predicted errors are tabulated for the representative unit problem of pressure equal to $1 \mathrm{~atm}$ and path length equal to $1.0 \mathrm{~m}$ per the error prediction procedure established in previous work [6]. The spectral error estimators are found individually for water vapor, carbon dioxide, the hydroxyl radical, and carbon monoxide, each with a mole fraction of 0.10 . The calculations are repeated for each $25 \mathrm{~cm}^{-1}$ band ranging from $25-10,000 \mathrm{~cm}^{-1}(200-1.00 \mu \mathrm{m})$. The calculations are also repeated for the six temperatures of 600,1000 , $1500,2000,2500$, and $3000 \mathrm{~K}$, resulting in a three-dimensional predicted error table with 4 species, 6 temperatures, and 399 bands. These are the same dimensions as the absorptivity tables.

With the absorption and uncertainty tables calculated, the uncertainty of a full simulation can be quantified. The method employed is a differencing scheme, wherein the physical domain is simulated for each spectral quadrature point using the RT method [Eq. (4)] and spectrally integrated using Eq. (6). The scheme is then repeated with the absorption tables perturbed by the error tables.
The resulting differences in the radiative heat flux are taken as the spectral uncertainty of the system as given by Eq. (12), where $U$ is the dimensionless uncertainty factor:

$$
U(s)=\frac{\Delta F(s)}{F_{0}(s)}-1
$$

\section{E. Computational Fluid Dynamics}

The input data for the radiative simulations codes are CFD simulation results. The CFD results are performed at the AFRL using the CFD++ code with a $k-\epsilon$ RANS solver. The chemical mechanism is a 22-species combustion model as developed by Wang and Laskin [23]. Further details of the mechanisms and solvers can be found in the original publication of the CFD simulations by Storch et al. [4].

The specific simulation of interest is intended to duplicate the conditions of a Mach 6.5 flight of the HIFiRE 2 scramjet with an equivalence ratio of 1.0 for a methane-ethylene fuel with a mass ratio 0.36-064 between the two fuels, respectively, and an oxidizer of air. The simulation is run with 1.4 million grid points for a quarter of the domain employing planes of symmetry at both the vertical and horizontal centerlines. The full physical domain can be seen in Fig. 2, with the quarter section simulation domain shown in gray.

\section{F. Experimental Setup}

The experimental emission measurements are made on the HDCR in the Arc-Heated Scramjet Test Facility (AHSTF) at NASA's Langley Research Center. The HDCR is constructed and operated to provide ground-test support for Flight 2 of the HIFiRE program using the same flowpath lines as the flight engine [24].

An optical diagnostics flange and attendant hardware are attached to the engine at the exit of the combustor. The primary function of the optical hardware is to execute tunable diode laser absorption measurements along 16 lines of sight across (and adjacent to) the combustor exit. Three diode lasers are sequentially tuned across multiple water spectral features in the neighborhood of $1.4 \mu \mathrm{m}$ $\left(7143 \mathrm{~cm}^{-1}\right)$ with the aim of determining the temperature and water concentration fields at the exit plane [9]. The absorption measurements are conducted in such a way that for every $1 \mathrm{~ms}$ of data collection approximately $100 \mu$ s of signal due just to the nascent hot gas emission are also collected, which is what is reported in the current work. The emission signals are captured using Indium Gallium Arsenide (InGaAs) photodetectors with a narrow spectral bandwidth of $1.1-1.8 \mu \mathrm{m}$ (5556-9091 $\left.\mathrm{cm}^{-1}\right)$. A silicon filter removes emission at wavelengths below $1.1 \mu \mathrm{m}\left(9091 \mathrm{~cm}^{-1}\right)$. (The filter is necessary to maintain good signal-to-noise ratios in the absorption measurements.) A photograph of the optical hardware attached to the HDCR is shown in Fig. 3. Sixteen photodetectors and associated transimpedance amplifiers are split between two electronics boxes, one positioned on the body (top) side of the combustor exit and one on the port (left) side of the combustor exit.

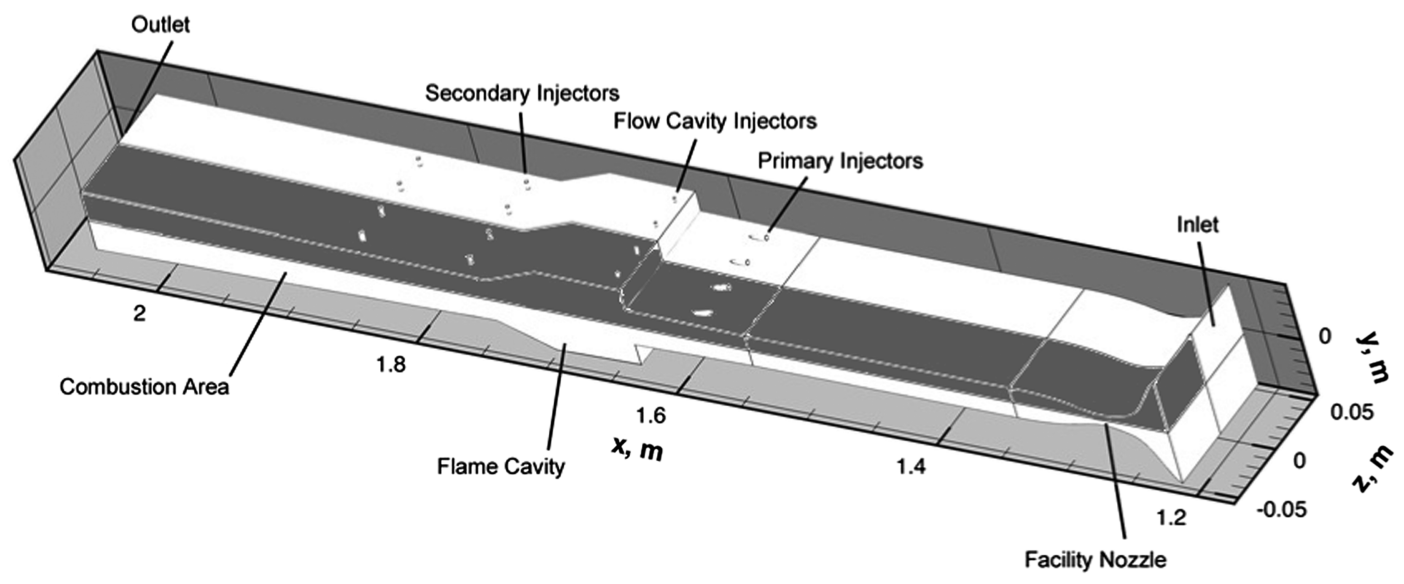

Fig. 2 HDCR domain: Illustration of the facility nozzle, flame holding cavity, injection ports, and outlet. Flow is from right to left. 


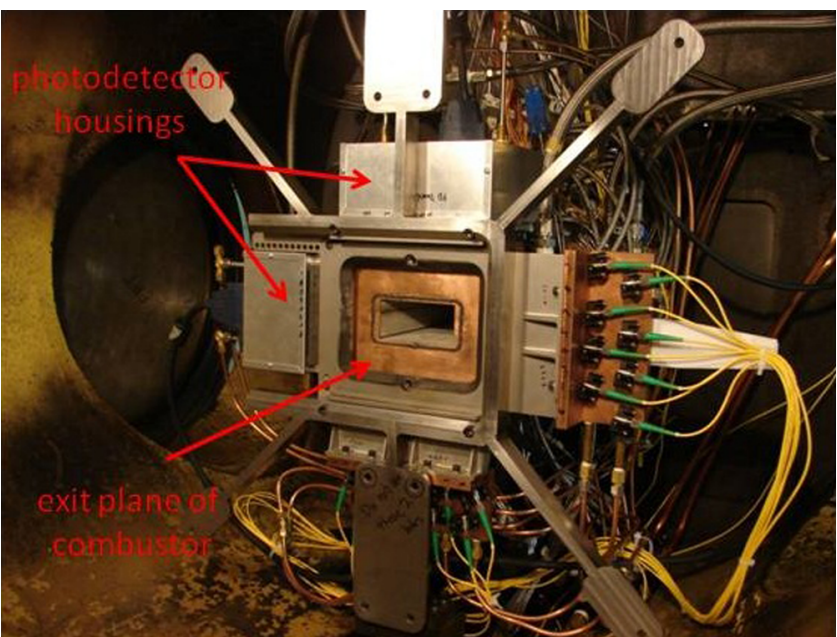

Fig. 3 Optical hardware mounted to the exit plane of the HDCR combustor. The 16 photodetectors used in the emission measurements are located in two housings as indicated. Flow is right to left.

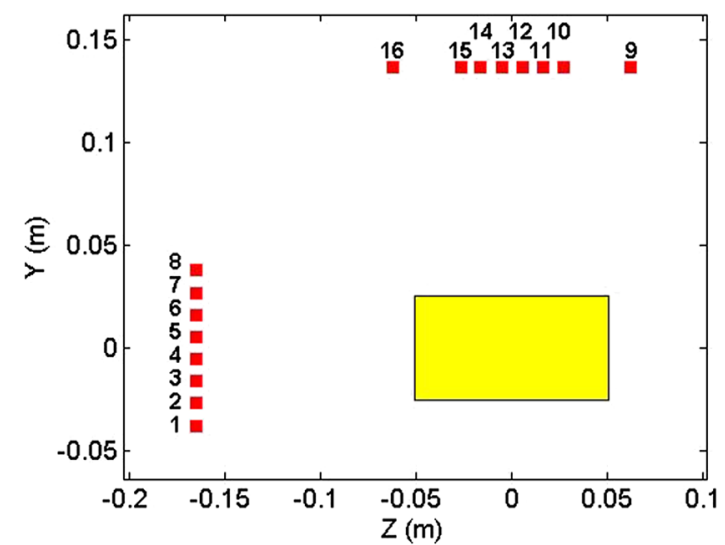

Fig. 4 Location of the emission photodetectors with respect to the exit of the combustor shown in the box. Flow is out of the page.

This arrangement permits collection of the emission from eight vertical views and eight horizontal views that share some common overlap with respect to their solid angles of emission collection. Figure 4 shows a scaled schematic of the location of the 16 individual photodetectors with respect to the combustor exit, which is $0.05 \mathrm{~m}$ tall and $0.10 \mathrm{~m}$ wide. Apertures located between the photodetectors and combustor exit limit the solid angle of light collection. Specific values of the light collection (field of view and sheet thickness) for each photodetector are noted in Table 1 . The photodetectors and all optics associated with the experiment are calibrated using a single tunable diode laser with a known power. The photodetector calibration procedure is given in Appendix A of the thesis by Crow [6].

\section{Results}

A brief description of the CFD simulations were originally presented in previous work [5]. Additionally, a comparison between experimental and simulated radiative heat flux measurements is provided.

The computational results for the simulation of a Mach 6.5 flight of the HIFiRE 2 scramjet with an equivalence ratio of 1.0 for a 0.36 0.64 methane-ethylene fuel are given in the paper by Crow et al. [25]. However, only the exit plane quantities are of interest in the current work and are given in Fig. 5 for temperature and water mole fraction. The CFD simulation data can be directly compared to experimental measurements taken on the HDCR presented in the paper by Brown et al. [9].

The results from the CFD simulation differ greatly from those of the experimental measurements. The experimental measurements suggest a hotter and higher water mole fraction than do the simulations. The temperature forms a hot ring around the edges in Fig. 5a, whereas the experiment shows no structure in Fig. 6a. The simulated water vapor mole fraction given in Fig. $5 \mathrm{~b}$ is significantly less evenly distributed than would be suggested by experimental results given in Fig. 6b. Large-eddy simulations (LESs) of the HDCR have shown better agreement with the overall structure of the flowfield than the RANS CFD simulations produce [6]. However, the boundary conditions of the LESs have shown less realistic flow temperatures than those measured on the HDCR.

As described earlier, thermal radiation over a narrow infrared band is measured in the exhaust nozzle of the experimental test rig. Using the setup on the HDCR in the AHSTF at NASA's Langley Research Center described previously, the infrared signal is measured from 1.1 to $1.8 \mu \mathrm{m}\left(5556-9091 \mathrm{~cm}^{-1}\right)$ and averaged over a $0.25 \mathrm{~s}$ window. The relative photodetector response is greater than $70 \%$ between 6061 and $9091 \mathrm{~cm}^{-1}$ ). Each reported signal is corrected for intrinsic electronic noise. Previous work has examined the photodetector responses in calibrated and uncalibrated manners [ $[\underline{5}, 6]$.

The calibrated data in previous works showed a good agreement between simulations and experiments for photodetectors placed within $1 \mathrm{~mm}$ of the silicon filter (sensors 1-9 and 16) used to protect the photodetectors. However, photodetectors with standoff distances in excess of $3 \mathrm{~mm}$ from the silicon filter (sensors 10-15) gave a calibrated spectral measurement significantly lower than the simulations predicted in previous works. One possible explanation for this discrepancy is the textured surface of the silicon window, which may redirect the narrow angles of incident thermal radiation. A correction factor is investigated to account for scattering effects.

A collimated infrared diode laser is employed identical to that of the photodetector power calibration setup [6]. The silicon filter is placed between the collimated laser and the individual photodetectors. The standoff distance from the silicon filter to the photodetector is varied. With a known Gaussian spread and a known photodetector area, the beam scattering angle can be estimated. A scattering model angle assumes that at every point of the beam incident to the silicon filter the collimated beam is converted from a column to a cone with a fixed angle. The tangent function of the predicted scattering angle multiplied by the standoff distance from the silicon window gives the effective linear increase in size of the radiation passing through the aperture, spreading the thermal radiation over a wider area, thus reducing the local intensity. The relative change in the effective area is employed as the correction factor for the experimental measurements. A mathematical description is given in Eq. (13):

$$
F_{\text {sens }}=\int_{0}^{A_{\text {sens }}} \int_{0}^{A_{\text {filt }}} \frac{F\left(A_{\text {filt }}\right)}{(w \tan (\omega))^{2} \pi} \mathrm{d} A_{\text {filt }} \mathrm{d} A_{\text {sens }} / A_{\text {sens }}
$$

Table 1 Position and field of view of photodetectors at the exhaust port

\begin{tabular}{lccccc}
\hline \hline $\begin{array}{l}\text { Sensor } \\
\text { number }\end{array}$ & Orientation & $\begin{array}{c}\text { Field of } \\
\text { view, deg }\end{array}$ & $\begin{array}{c}\text { Sheet thickness, } \\
\text { deg }\end{array}$ & $\begin{array}{c}Y \text { position, } \\
\text { m }\end{array}$ & $\begin{array}{c}Z \text { position, } \\
\mathrm{m}\end{array}$ \\
\hline 1 & Horizontal & 48.3 & 3.9 & -0.038 & 0.168 \\
2 & Horizontal & 45.2 & 3.9 & -0.027 & 0.168 \\
3 & Horizontal & 23.9 & 3.9 & -0.016 & 0.174 \\
4 & Horizontal & 23.9 & 3.9 & -0.005 & 0.174 \\
5 & Horizontal & 23.9 & 3.9 & 0.005 & 0.174 \\
6 & Horizontal & 23.9 & 3.9 & 0.016 & 0.174 \\
7 & Horizontal & 45.2 & 3.9 & 0.027 & 0.168 \\
8 & Horizontal & 49.0 & 3.9 & 0.038 & 0.168 \\
9 & Vertical & 49.8 & 4.0 & 0.139 & 0.062 \\
10 & Vertical & 20.1 & 4.0 & 0.146 & 0.027 \\
11 & Vertical & 20.2 & 4.0 & 0.146 & 0.016 \\
12 & Vertical & 21.4 & 4.0 & 0.146 & 0.005 \\
13 & Vertical & 21.4 & 4.0 & 0.146 & -0.005 \\
14 & Vertical & 20.2 & 4.0 & 0.146 & -0.016 \\
15 & Vertical & 20.2 & 4.0 & 0.146 & -0.027 \\
16 & Vertical & 50.0 & 4.0 & 0.139 & -0.062 \\
\hline \hline
\end{tabular}




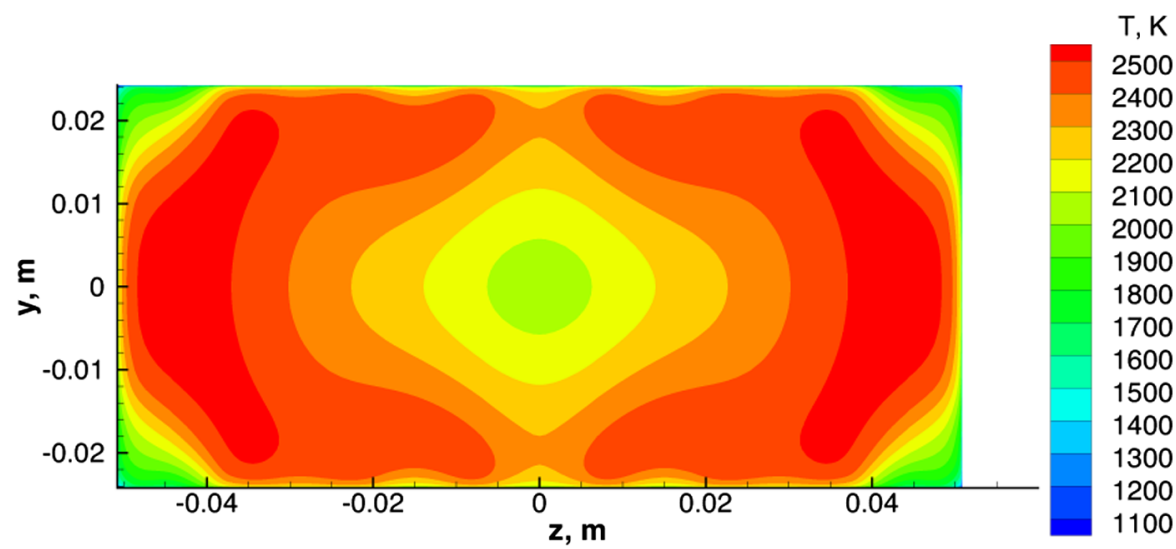

a)

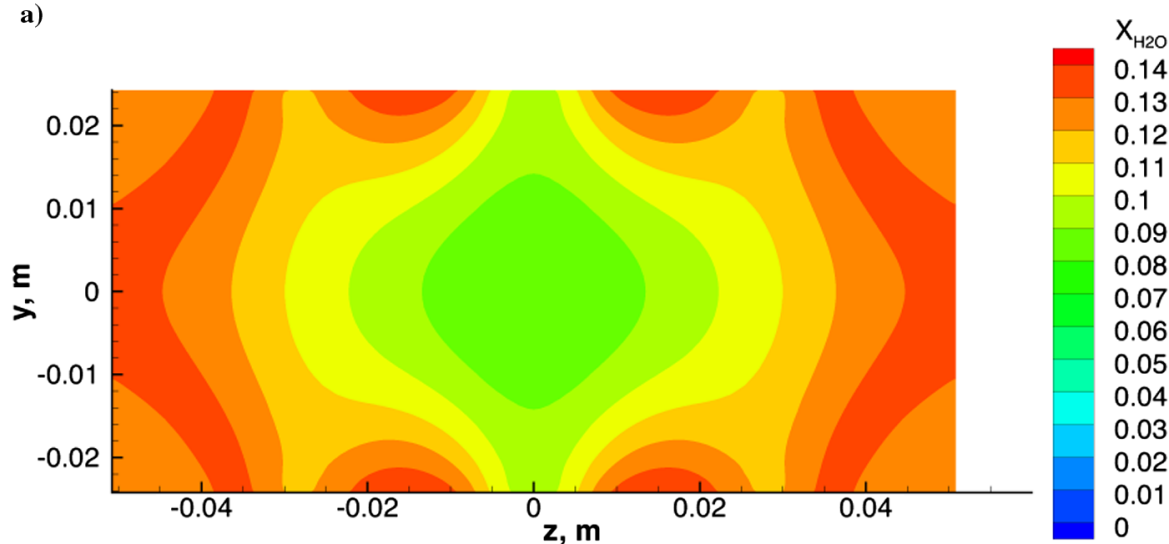

b)

Fig. 5 Exit plane profiles for a CFD RANS simulation of the HDCR experiments for the a) temperature and b) water vapor mole fraction. Flow is out of the page.

The experimental conditions are simulated using a RT method with a narrow-band spectral model. The physical domains of the simulations are intended to duplicate the exact field of view for each sensor, given the position of the sensor and the geometry of the mounting hardware. The details are given in Table 1 . The simulations employ an angular resolution of $0.5 \mathrm{deg}$ along the solid-angle field of view and a spatial resolution of $0.5 \mathrm{~mm}$ along each trace path, which is found to be completely converged given the current test case. The spectral model employs 138 bands ranging in frequencies from 5550 to $9100 \mathrm{~cm}^{-1}$ with an evenly distributed spectral resolution of $25 \mathrm{~cm}^{-1}$. This spectral range corresponds to the combined effects of the physical sensor response function and the silicon filtering window. The resulting predictions are given in Fig. 7. Because of the limited field of view ranging from 0.02 to $0.06 \mathrm{sr}$ and spectral range of the simulations only capturing 1.9 to $2.4 \%$ of spectrally resolved radiative heat flux, these predictions are about $0.05 \%$ of the total radiative heat flux to the location of interest.

In addition to the spectral modeling error, the spectral database error coming from the original HiTemp spectral database also needs to be included. The spectral modeling error employed is calculated over the $1 \mathrm{~m}$ path length as described previously. The spectral database error is accounted for by adding a factor of $20 \%$ to the existing spectral model error, resulting in a value of $E=0.20$. The spectral model is perturbed by the combined error as in Eq. (10), in order to determine the combined spectral uncertainty. This error is shown in Fig. 7

In Fig. 7, the experimental measurements are given with individual symbols and corresponding error bars, as each measurement is taken individually, and the factors affecting the certainty of the measurement may vary from detector to detector. The simulation results are given as correlated curves because all simulations use an identical spectral model. Therefore, any error in the spectral model would appear in all simulations. As such, all simulations would either be perturbed high or low due to spectral uncertainty but never with

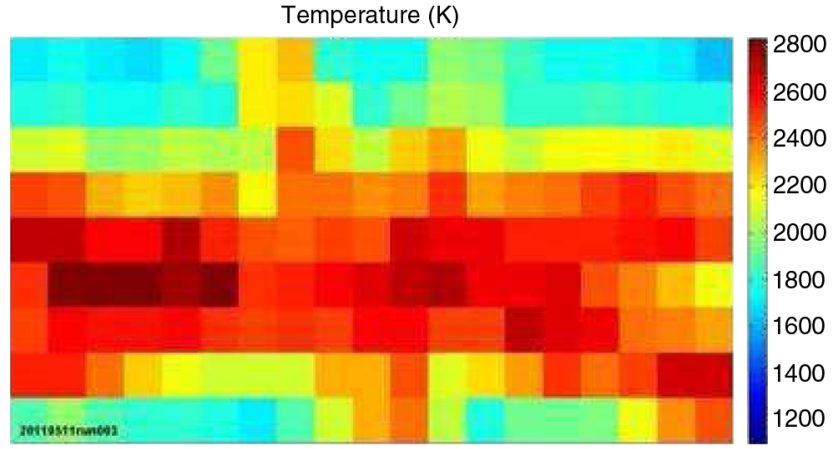

a)

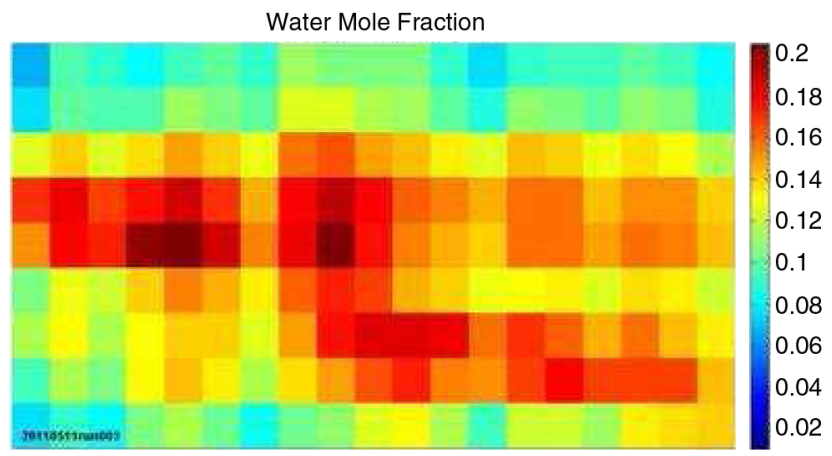

b)

Fig. 6 Exit plane profiles measured from the HDCR experiments for the a) temperature and b) water vapor mole fraction. Flow is out of the page. Measurements taken by Brown et al. [9]. 


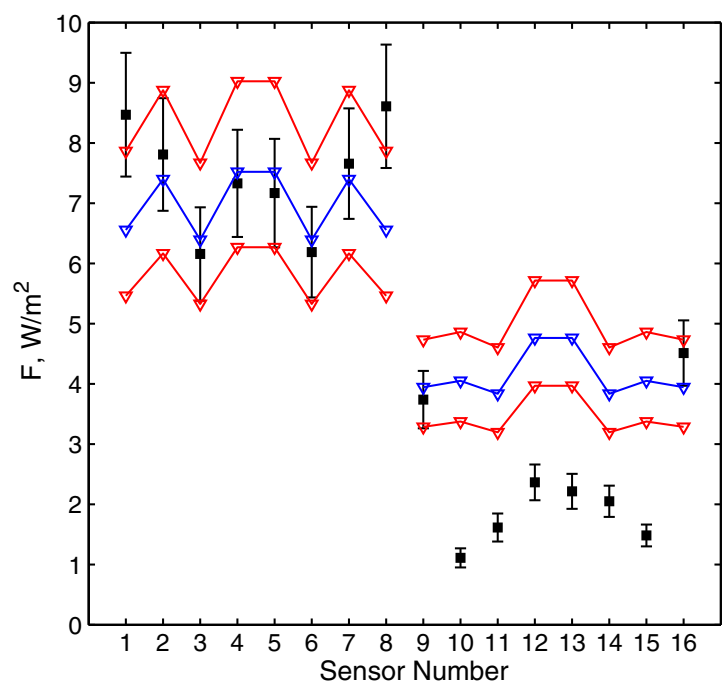

a)

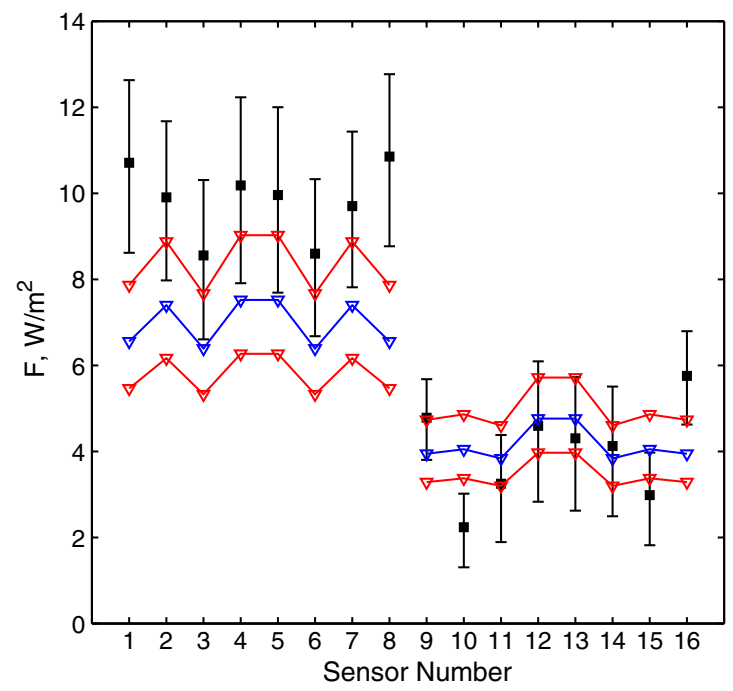

b)

Fig. 7 Exhaust port heat flux data: a) without scatting correction and b) with scattering correction.

a mixture of results. In Fig. 7, the high and low limits are given for the computational predictions as additional curves above and below the nominal values.

In Fig. 7a, the simulation results have agreement within experimental uncertainty for sensors 9 and 16 of the vertical photodetectors and for sensors 2 through 7 of the horizontal photodetectors. The vertical sensors 10-15 show similar relative trends between experiment and simulation, but they do not overlap in any absolute sense. Sensors 1 and 8 show an agreement with the highest simulations predictions fitting into the experimental error bars, but this overlap does not match the trend presented by sensors $2-7$.

Incorporating the optical scattering correction due to silicon into the experimental measurement in Fig. 7b, both the magnitude and the error bars increase. The experimental error bars increase from a maximum of $+14 /-12 \%$ in Fig. 7 for unscatterd predictions, part a, to a maximum value of $+23 /-21 \%$ for sensors with a $1 \mathrm{~mm}$ standoff distance from the silicon filter or to $+41 /-35 \%$ for sensors with a $3 \mathrm{~mm}$ standoff distance (sensors 10-15) from the silicon filter, part $b$.

The experimental predictions of sensors 1 and 8 are now no longer overlapping with the simulation predictions. The experimental measurements of sensors 9 and 11-15 now overlap the simulation predictions. Only sensor 10 has not moved into the simulation range. The remaining sensors still overlap the simulations predictions, but now they have slightly higher values.
The explanation as to why only certain sensor simulations correlate with experiments may lie in the flowfield. The experimental flowfield is characterized for water vapor mole fraction and temperature by Brown et al. [9]. The temperature and water vapor distribution do not closely match those predicted by the CFD RANS predictions as given in Figs. 5 and 6 .

The differences between the simulated and experimental flowfields can account for differences in the radiative heating. If the flows are equal in combustion completion and heat release, then their differences would mainly be due to the spatial distribution of the flowfield quantities. Sensors that view a large amount of the flowfield would be unaffected by the changes in distribution. Because of their orientations and fields of view, sensors $1-8,9$, and 16 view almost all of the flow. Although sensors 3-6 have a narrower field of view than sensors 1, 2, 7, and 8, they are oriented at the narrower side of the flowfield. Therefore, they are still able to capture the majority of the flow. It is hypothesized that the ability of the sensors to see the whole flowfield and not just a local area is the reason the simulations of these sensors yield results close to the experimental measurements. The edge of the flow has a significant amount of mixing with the external surrounding air, which is not accounted for in the simulations as they end at the exit plane. Thus, the sensors may see more radiative material in their fields of view than the simulation can predict, resulting in the simulations underpredicting the thermal radiative heat flux for sensors $1,8,9$, and 16. Sensors $10-15$ only view a narrow column. As such, any small shift in flowfield distribution causes a significant change in the radiative material in their fields of view. Thus, the simulation predictions do not match the experimental measurements as closely.

\section{Conclusions}

Flow in the HIFiRE 2 combustor was modeled using a $k-\epsilon$ RANS simulation.

The thermal radiation at the exhaust port was measured experimentally using an array of infrared photodetectors on the HDCR in the AHSTF at NASA's Langley Research Center. The emissions at these same measurement locations were also simulated using a ray-tracing radiative heat transfer code. Currently, these values are the first published direct quantitative measurements of radiative heat transfer in a hydrocarbon scramjet and the first of a scramjet setup based on a flight experiment. The experiments agreed with the simulations for the photodetectors with wider fields of view, suggesting that the overall CFD simulations and radiative simulation methods are adequate.

Future work includes the incorporation of LES simulations. The LES simulation has been shown in previous studies of the HDCR to have a closer agreement with the features of the experimental flowfields [6]. Additionally, the temporal and spatial variations caused by turbulent fluctuation have been shown to greatly increase the magnitude of the thermal radiative heat flux in the HDCR. Furthermore, studies support a possible increase in thermal radiative heat transfer due to temporal variations [26,27]. Finally, more stringent controls to over the surface quality of the photodetector filters will be implemented in future measurements to eliminate the need for scattering correction factors.

\section{Acknowledgments}

This material is based upon work supported by the Department of Energy (National Nuclear Security Administration) under award number NA28614. The experimental work was supported in part by Advanced Propulsion Test Technology (Ed Tucker, Executing Agent) and the U.S. Air Force Office of Scientific Research (John Schmisseur, Program Manager). This report was prepared as an account of work sponsored by an agency of the United States Government. Neither the United States Government nor any agency thereof, nor any of their employees, makes any warranty, express or implied, or assumes any legal liability or responsibility for the accuracy, completeness, or usefulness of any information, apparatus, product, or process disclosed or represents that its use would not infringe privately owned rights. Reference herein to any specific 
commercial product, process, or service by trade name, trademark, manufacturer, or otherwise does not necessarily constitute or imply its endorsement, recommendation, or favoring by the United States Government or any agency thereof. The views and opinions of authors expressed herein do not necessarily state or reflect those of the United States Government or any agency thereof.

\section{References}

[1] Crow, A. J., Boyd, I. D., and Terrapon, V. E., "Radiation Modeling of a Hydrogen-Fueled Scramjet," 42nd AIAA Thermophysics Conference, AIAA Paper 2011-3769, June 2011.

[2] Jackson, K. R., Gruber, M. R., and Barhorst, T., "The HIFiRE Flight 2 Experiment: An Overview and Status Update," AIAA Paper 20095029, 2009.

[3] Jackson, K. R., Gruber, M. R., and Buccellato, S., "HIFiRE Flight 2 Overview and Status Update 2011," 17th AIAA International Space Planes and Hypersonic Systems and Technologies Conference, AIAA Paper 2011-2202, April 2011.

[4] Storch, A. M., Bynum, M., Liu, J., and Gruber, M. R., "Combustor Operability and Performance Verification for HIFiRE Flight 2," 17th AIAA International Space Planes and Hypersonic Systems and Technologies Conference, AIAA Paper 2011-2249, April 2011.

[5] Crow, A. J., Boyd, I. D., Brown, M. S., and Liu, J., "Thermal Radiative Analysis of the HIFiRE-2 Scramjet Engine," 43rd AIAA Thermophysics Conference, AIAA Paper 2012-2751, June 2012.

[6] Crow, A. J., "Uncertainty Quantification of Thermal Radiation in Supersonic Combustion Chambers," Ph.D. Thesis, Univ. of Michigan, Ann Arbor, MI, May 2013.

[7] Rothman, L. S., Gordon, I. E., Dothe, R. J. B. H., Gamache, R. R., Goldman, A., Perevalov, V. I., Tashkun, S. A., and Tennyson, J., "HiTemp, The High-Temperature Molecular Spectroscopic Database," Journal of Quantitative Spectroscopy and Radiative Transfer, Vol. 111, No. 15,2010 , pp. 2139-2150. doi:10.1016/j.jqsit.2010.05.001

[8] Liu, L., Tan, H., and He, Z., "Inverse Radiation Problem of Source Term in Three-Dimensional Complicated Geometric Semitransparent Media," International Journal of Thermal Sciences, Vol. 40, No. 6, 2001, pp. 528-538. doi:10.1016/S1290-0729(01)01241-8

[9] Brown, M. S., Herring, G. C., Cabell, K., Hass, N., Barhorst, T. F., and Gruber, M. R., "Optical Measurements at the Combustor Exit of the HIFiRE 2 Ground Test Engine," 50th AIAA Aerospace Sciences Meeting Including the New Horizons Forum and Aerospace Exposition, AIAA Paper 2012-0857, Jan. 2012.

[10] Reed, R. A., and Slack, M. W., "Infrared Measurements of a Scramjet Exhaust,” Langley Research Center TR-CR-3242, Jan. 1980.

[11] Hopkins, H. B., Konopka, W., and Leng, J., "Validation of Scramjet Exhaust Simulation Technique," Langley Research Center TR-CR2688, June 1976.

[12] Liu, J., and Gruber, M. R., "Preliminary Preflight CFD Study on the HIFiRE Flight 2 Experiment," 17th AIAA International Space Planes and Hypersonic Systems and Technologies Conference, AIAA Paper 2011-2204, April 2011.

[13] Pope, S. B., Turbulent Flows, Cambridge Univ. Press, New York, 2000, pp. 383-384.
[14] Modest, M. F., Radiative Heat Transfer, 2nd ed., Academic Press, New York, 2003, pp. 655-661.

[15] Coelho, P. J., Perez, P., and El Hafi, M., "Benchmark Numerical Solutions for Radiative Heat Transfer in Two-Dimensional Axisymmetric Enclosures with Nongray Sooting Media," Numerical Heat Transfer, Part B, Vol. 43, No. 5, 2003, pp. 425-444. doi: $10.1080 / 713836240$

[16] Planck, M., The Theory of Heat Radiation, 2nd ed., Dover, New York, 1991, pp. 167-189.

[17] Liou, K. N., An Introduction to Atmospheric Radiation, 2nd ed., Academic Press, New York, 2002, pp. 90-97, 260-274.

[18] Wang, A., and Modest, M. F., "High-Accuracy, Compact Database of Narrow-Band k-Distributions for Water Vapor and Carbon Dioxide," Journal of Quantitative Spectroscopy and Radiative Transfer, Vol. 93, Nos. 1-3, 2005, pp. 245-261. doi:10.1016/j.jgsrt.2004.08.024

[19] Pal, G., and Modest, M. F., "A Narrow Band-Based Multiscale Multigroup Full-Spectrum k-Distribution Method for Radiative Transfer in Nonhomogeneous Gas-Soot Mixtures," Journal of Heat Transfer, Vol. 132, No. 2, Feb. 2010, pp. 023307-1-023307-9.

[20] Modest, M. F., and Riazzi, R. J., "Assembly of Full-Spectrum kDistributions from a Narrow-Band Database; Effects of Mixing Gases, Gases and Nongray Absorbing Particles, and Mixtures with Nongray Scatterers in Nongray Enclosures," Journal of Quantitative Spectroscopy and Radiative Transfer, Vol. 90, No. 2, 2005, pp. 169-189. doi:10.1016/j.jgsrt.2004.03.007

[21] Ludwig, C. B., Malkmus, W., Reardon, J. E., and Thomson, J. A. L., "Handbook of Infrared Radiation from Combustion Gases," NASA TRSP-3080, 1973.

[22] Irvine, A., "High Fidelity Radiative Thermal Transport Simulations of a Scramjet Propulsion System," Ph.D. Thesis, Univ. of Michigan, Ann Arbor, MI, 2013.

[23] Wang, H., and Laskin, A., "A Comprehensive Kinetic Model of Ethylene and Acetylene Oxidation at High Temperature," Internal Report for an AFOSR New World Vista Program, U.S. Air Force Office of Scientific Research, 1998.

[24] Cabell, K., Hass, N. E., Storch, A. M., and Gruber, M. R., "HIFiRE Direct-Connect Rig (HDCR) Phase 1 Scramjet Test Results from the NASA Langley Arc-Heated Scramjet Test Facility," 17th AIAA International Space Planes and Hypersonic Systems and Technologies Conference, AIAA Paper 2011-2248, April 2011.

[25] Crow, A. J., Boyd, I. D., and Terrapon, V. E., "Radiation Modeling of a Hydrogen Fueled Scramjet," Journal of Thermophysics and Heat Transfer, Vol. 27, No. 1, 2013, pp. 11-21. doi:10.2514/1.T3751

[26] Coelho, P. J., "Numerical Simulation of the Interaction Between Turbulence and Radiation in Reactive Flows," Progress in Energy and Combustion Science, Vol. 33, No. 4, Jan. 2007, pp. 311-383. doi:10.1016/j.pecs.2006.11.002

[27] Coelho, P. J., "Turbulence-Radiation Interaction: From Theory to Application in Numerical Simulations," Journal of Heat Transfer, Vol. 134, No. 3, March 2012, pp. 031001-1-031001-13. doi: $10.1115 / 1.4005130$

G. Richards

Associate Editor 